\title{
100. Note on Locally Definable Classes of Structures
}

\author{
By Takao Kashiwagi \\ Yamaguchi University \\ (Comm. by Kenjiro Shoda, M. J. A., Sept. 12, 1974)
}

In this note we shall study certain generalizations of the following property (\#) for a class $K$ of structures:

(\#) A structure $\mathfrak{A}$ is in $K$ whenever each finite relative partial substructure of $\mathfrak{A}$ defined by each finite number of operations and relations can be embedded in some structure in $K$.

This property (\#) was introduced by Mal'cev ([2] and [3]), and he called a class having the property (\#) locally definable. On the property (\#) the following is known (Tarski [6] and Mal'cev [4; p. 138]):

(*) A class $K$ is universal if and only if $K$ has the property (\#).

Tarski [7] gave two analogous theorems. One is for an infinitary language without operation symbols and the other is for an infinitary language without relation symbols. We shall make the similar investigation for an infinitary language $L_{\alpha \beta}$ with an arbitrary number of finitary operation and relation symbols. We introduce a notion of $[\alpha, \beta]$-local definability as an analogue of local definability. Our main theorem below, which is a generalization of the theorems of Tarski ([7; Theorems 1 and 2]), shows that every $[\alpha, \beta]$-locally definable class can be characterized as a class defined by a set of universal sentences in prenex form of the infinitary language $L_{\alpha \beta}$. A generalization of (*) is stated in Corollary of this theorem.

There are two similar works for classes of algebras in [5] and for classes of relational systems in [1]. The former ([5; Theorem 2 ((i) $\Longleftrightarrow$ (ii))] follows immediately from our theorem. But it seems that the latter does not follow from our results, because [1] deals with classes defined by universal sentences of $L_{\alpha \beta}$ which are not necessarily in prenex form.

The letters $\lambda, \mu, \nu, \xi, \zeta$ will be used to denote ordinals, and $\alpha, \beta$ will be used to denote cardinals (initial ordinals). The cardinality of a set $A$ is denoted by $|A| . \quad \mathfrak{A}=\left\langle A ;\left\{f_{A}: f \in F\right\},\left\{r_{A}: r \in R\right\}\right\rangle$ is called a structure, if $A$ is a nonvoid set, and there are maps $n: F \rightarrow \omega$ and $m: R \rightarrow \omega$ such that for $f \in F, f_{A}$ is an $n(f)$-ary operation on $A$ and for $r \in R, r_{A}$ is an $m(r)$-ary relation on $A . \quad A$ is called the universe of $\mathscr{U}$. The sequence $\tau=\langle F, n, R, m\rangle$, uniquely determined by $\mathfrak{A}$, is called the similarity type of $\mathfrak{A}$. The type $\tau$ is fixed throughout this note. Capital German letters denote structures and the corresponding capital Roman 
letter denotes the universe of the structure.

For each pair of infinite cardinals $\alpha, \beta$ with $\alpha \geqq \beta$, we can define the infinitary language $L_{\alpha \beta}$ with equality and with the set $F$ of operation symbols and the set $R$ of relation symbols which corresponds to the similarity type $\tau$. Throughout this note, we assume that the cardinal $\alpha$ is regular. For any formula $\phi$ of $L_{\alpha \beta}$, we may write $\phi\left(v_{\zeta}: \zeta<\nu\right)$ to indicate that the free variables of $\phi$ are members of $\left\{v_{\zeta}: \zeta<\nu\right\}$. For any structure $\mathfrak{A}$ and elements $a_{\zeta}, \zeta<\nu$, of $A$, $\mathfrak{A} \vDash \phi\left[a_{\zeta}: \zeta<\nu\right]$ indicates that $\phi$ is satisfied in $\mathscr{A}$ when $a_{\zeta}$ is assigned to $v_{\xi}, \zeta<\nu$. Let $\left\{c_{\xi}: \xi<\mu\right\}$ be a set of new constants. Then we denote by $L_{\alpha \beta}(\mu)$ the language obtained from $L_{\alpha \beta}$ by adding the constants $c_{\xi}, \xi<\mu$. Let $\mathfrak{U}$ be a structure and let $a=\left\langle a_{\xi}: \xi\langle\mu\rangle\right.$ be a sequence of elements in $A$. Then we denote by ( $\mathfrak{A}, a)$ the structure for $L_{\alpha \beta}(\mu)$ which is obtained from $\mathfrak{A}$ by interpreting each $c_{\xi}$ by $a_{\xi}$. Let $F^{\prime}$ and $R^{\prime}$ be subsets of $F$ and $R$ respectively. We denote by $L_{\alpha \beta} \Gamma\left(F^{\prime}, R^{\prime}\right)$ the language obtained from $L_{\alpha \beta}$ by omitting the nonlogical symbols not contained in $F^{\prime} \cup R^{\prime}$. The reduct of $\mathscr{A}$ to $L_{\alpha \beta} \Gamma\left(F^{\prime}, R^{\prime}\right)$ is denoted by $\mathfrak{x} \Gamma\left(F^{\prime}, R^{\prime}\right)$. We denote by $S\left(\mu, F^{\prime}, R^{\prime}\right)$ the set consisting of all atomic sentences of $L_{\alpha \beta}(\mu) \Gamma\left(F^{\prime} \cup\left\{c_{\xi}: \xi<\mu\right\}, R^{\prime}\right)$ and their negations. Now we define the sets $D_{\left(F^{\prime}, R^{\prime}\right)}(\mathfrak{H}, a)$ and $d_{\left(F^{\prime}, R^{\prime}\right)}(\mathfrak{A}, a)$ as follows:

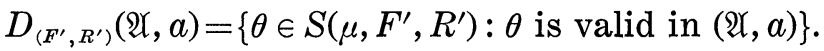

$$
\begin{aligned}
& d_{\left(F^{\prime}, R^{\prime}\right)}(\mathfrak{U}, a)=\left\{\theta \in D_{\left(F^{\prime}, R^{\prime}\right)}(\mathfrak{A}, a): \theta\right. \text { is of the form } \\
& \left.c_{\xi}=c_{\xi}, \quad\right\rceil c_{\xi}=c_{\xi}, \quad f\left(c_{\xi_{1}}, \cdots, c_{\xi_{n(f)}}\right)=c_{\zeta}, \\
& \rceil f\left(c_{\xi_{1}}, \cdots, c_{\xi_{n}(f)}\right)=c_{\xi}, \quad r\left(c_{\xi_{1}}, \cdots, c_{\xi_{m}(r)}\right) \\
& \text { or }\rceil r\left(c_{\xi_{1}}, \cdots, c_{\xi_{m(r)}}\right) \text {, where } f \in F^{\prime} \text {, } \\
& \text { and } r \in R^{\prime} \text {. }
\end{aligned}
$$

The following lemma can be easily verified:

Lemma. Assume that $\omega \leqq \beta<\alpha$ or $\omega<\beta \leqq \alpha$. If $\mu<\beta$ and $\left|F^{\prime} \cup R^{\prime}\right|$ $<\alpha$, then $\left|D_{\left(F^{\prime}, R^{\prime}\right)}(\mathfrak{A}, a)\right|<\alpha$.

Let $K$ be a class of structures. $K$ is said to be $[\alpha, \beta]$-(resp. $(\alpha, \alpha)$-) locally definable if, a structure $\mathfrak{A}$ is in $K$ whenever for any $a \in A^{\mu}$ with $\mu<\beta$ (resp. $\mu<\alpha)$ and any $F^{\prime} \cup R^{\prime} \subset F \cup R$ with $\left|F^{\prime} \cup R^{\prime}\right|<\alpha$, there is a structure $\mathfrak{B}$ in $K$ such that $(\mathfrak{B}, b)$ is a model of $D_{\left(F^{\prime}, R^{\prime}\right)}(\mathfrak{A}, a)$ (resp. $\left.d_{\left(F^{\prime}, R^{\prime}\right)}(\mathfrak{A}, a)\right)$ for some $b \in B^{\mu}$.

Note that " $(\omega, \omega)$-locally definable" is equivalent to "locally definable" in the sense of Mal'cev, and that every $(\alpha, \alpha)$-locally definable class is $[\alpha, \alpha]$-locally definable, and conversely if $\alpha \neq \omega$.

Let $\Sigma$ be a set of sentences of $L_{\alpha \beta}$. The class of all models of $\Sigma$ is denoted by $M(\Sigma)$.

Theorem. If $\omega \leqq \beta<\alpha$ or $\omega<\beta \leqq \alpha$, then the following two conditions for a class $K$ of structures are equivalent:

(1) $K$ is $[\alpha, \beta]$-locally definable; 
(2) $K=M(\Sigma)$ for some set $\Sigma$ of universal sentences of $L_{\alpha \beta}$ which are written in prenex form.

Proof. $\quad(1) \Rightarrow(2)$. Let $\Sigma$ be the set of all universal sentences in prenex form of $L_{\alpha \beta}$ that are valid in every structure in $K$. Then it is clear that $K \subset M(\Sigma)$. We shall prove that $M(\Sigma) \subset K$. Suppose $\mathfrak{A} \in M(\Sigma)$. Now let $a \in A^{\mu}$ with $\mu<\beta$ and $F^{\prime} \cup R^{\prime} \subset F \cup R$ with $\left|F^{\prime} \cup R^{\prime}\right|<\alpha$. Then by Lemma, the following universal sentence $\phi$ in prenex form of $L_{\alpha \beta}$ can be defined:

$$
\left.\phi \equiv \forall v_{0} \cdots \forall v_{\xi} \cdots{ }_{\xi<\mu} \vee\{\rceil \theta^{\prime}: \theta \in D_{\left(F^{\prime}, R^{\prime}\right)}(\mathfrak{Y}, a)\right\}
$$

where $\theta^{\prime}$ is the formula of $L_{\alpha \beta}$ obtained from $\theta$ by replacing each constant $c_{\xi}$ by the variable $v_{\xi}$. Since it is clear that $\phi \notin \Sigma$, there is a structure $\mathfrak{B}$ in $K$ such that $\phi$ is not valid in $\mathfrak{B}$. Hence there is a sequence $b=\left\langle b_{\xi}: \xi\langle\mu\rangle \in B^{\mu}\right.$ such that $\mathfrak{B} \vDash \wedge\left\{\theta^{\prime}: \theta \in D_{\left(F^{\prime}, R^{\prime}\right)}(\mathfrak{A}, a)\right\}\left[b_{\xi}: \xi\right.$ $<\mu]$, and so $(\mathfrak{B}, b)$ is a model of $D_{\left(F^{\prime}, R^{\prime}\right)}(\mathfrak{U}, a)$. Hence by (1), $\mathfrak{X}$ is in $K$. Therefore $K=M(\Sigma)$.

(2) $\Rightarrow(1)$. Let $\mathfrak{A}$ be a structure. We assume that

( ६ ) for any $a \in A^{\mu}$ with $\mu<\beta$ and any $F^{\prime} \cup R^{\prime} \subset F \cup R$ with

$\left|F^{\prime} \cup R^{\prime}\right|<\alpha$, there is a structure $\mathfrak{B}$ in $K$ such that

$(\mathfrak{B}, b)$ is a model of $D_{\left(F^{\prime}, R^{\prime}\right)}(\mathfrak{A}, a)$ for some $b \in B^{\mu}$.

We shall prove that if $\phi \in \Sigma$ then $\mathfrak{U}$ is a model of $\phi$. We may assume that $\phi$ is of the following form:

$$
\phi \equiv \forall v_{0} \cdots \forall v_{\xi} \cdots{ }_{\xi<\nu<\beta} \psi\left(v_{\xi}: \xi<\nu\right)
$$

by suitably changing the variables. It suffices to prove that $\mathfrak{A} \vDash \psi\left[a_{\xi}: \xi\right.$ $\left\langle\nu\right.$ ] for any sequence $a=\left\langle a_{\xi}: \xi<\nu\right\rangle \in A^{\nu}$. Let $F^{\prime}$ and $R^{\prime}$ be the sets of all operation symbols and all relation symbols appearing in $\phi$. Then $\left|F^{\prime} \cup R^{\prime}\right|<\alpha$, because $\alpha$ is regular. Now assume that $\alpha=\left\langle\alpha_{\xi}: \xi\langle\nu\rangle\right.$ is any sequence in $A^{\nu}$. Then by the assumption $(\xi)$ there is a structure $\mathfrak{B}$ in $K$ such that $(\mathfrak{B}, b)$ is a model of $D_{\left(F^{\prime}, R^{\prime}\right)}(\mathfrak{A}, a)$ for some $b=\left\langle b_{\xi}: \xi<\nu\right\rangle$ $\in B^{\nu}$. We let $\mathfrak{B}_{0}$ be the substructure of $\mathfrak{B} \Gamma\left(F^{\prime}, R^{\prime}\right)$ which is generated by $\left\{b_{\xi}: \xi<\nu\right\}$. Since $\mathfrak{B}$ is in $K=M(\Sigma), \mathfrak{B} \vDash \psi\left[b_{\xi}: \xi<\nu\right]$. By the choice of $F^{\prime} \cup R^{\prime}, \mathfrak{B}_{0} \vDash \psi\left[b_{\xi}: \xi<\nu\right]$. Since $(\mathfrak{B}, b)$ is a model of $D_{\left(F^{\prime}, R^{\prime}\right)}(\mathfrak{Y}, a)$, the mapping $h: b_{\xi} \rightarrow a_{\xi}, \xi<\nu$, can be extended to an isomorphism from $\mathfrak{B}_{0}$ onto $\mathfrak{U}_{0}$, where $\mathfrak{U}_{0}$ is the substructure of $\mathfrak{U} \Gamma\left(F^{\prime}, R^{\prime}\right)$ generated by $\left\{a_{\xi}: \xi<\nu\right\}$. Hence $\mathfrak{A}_{0} \vDash \psi\left[a_{\xi}: \xi<\nu\right]$, and hence $\mathfrak{X} \vDash \psi\left[a_{\xi}: \xi<\nu\right]$. Therefore $\mathfrak{A}$ is a model of $\phi$, and therefore $\mathfrak{U} \in K=M(\Sigma)$. This completes the proof.

Corollary. The following two conditions for a class $K$ of structures are equivalent:

(1) $K$ is $(\alpha, \alpha)$-locally definable;

(2) $K=M(\Sigma)$ for some set $\Sigma$ of universal sentences of $L_{\alpha \alpha}$.

Proof. If $\alpha \neq \omega$, then the assertion is immediately obtained from Theorem. If $\alpha=\omega$, then it is obtained from Theorem 1 in $[4 ; \mathrm{p} .138]$. 


\section{References}

[1] E. G. K. Lopez-Escobar: Universal formulas in the infinitary language $L_{\alpha \beta}$. Bull. de l'Acad. Polon. des Sci. math. astr. phys., 13, 383-388 (1965).

[2] A. I. Mal'cev: Quasiprimitive classes of abstract algebras (in Russian). Dokl. Akad. Nauk SSSR, 108, 187-189 (1956).

[3] - : Several remarks on quasivarieties of algebraic systems (in Russian). Algebra i Logika Sem., 5, 3-9 (1966).

[4] A. I. Mal'cev: Algebraic Systems. Springer (1973).

[5] G. Sabbagh: On properties of countable character. Bull. Austral. Math. Soc., 4, 183-192 (1971).

[6] A. Tarski: Contributions to the theory of models. I. Indag. Math., 16, 572581 (1954).

[7] - Remarks on predicate logic with infinitely long expressions. Colloq. Math., 6, 171-176 (1958). 